\title{
LA CONSTRUCCIÓN DEL CONCEPTO DE MERCANCÍA EN LA TEORÍA ECONÓMICA
}

\author{
Alejandro Nadal* \\ El Colegio de México
}

\section{INTRODUCCIÓN}

LA CONSTRUCCIÓN DEL CONCEPTO DE mercancía constituye una tarea fundamental para la teoría económica: se trata del concepto del objeto de la disciplina, concepto indispensable para el desarrollo de todo el discurso económico. ${ }^{1}$ Constituyendo la teoría económica una teoría sobre la sociedad, este concepto se refiere a la expresión objetiva de relaciones sociales a través de magnitudes numéricas. En efecto, el bien económico "mercancía" es objeto de una serie de operaciones de intercambio, concebidas como relaciones de equivalencia, a través de las cuales se lleva a cabo una operación de medida que permite asociar un coeficiente numérico ("precio") a cada bien económico. Es en tanto mercancías que los bienes entran en la rela-

- El autor agradece los comentarios críticos y la valiosa ayuda proporcionada por el profesor Carlos Salas Páez en la elaboración de este ensayo. Desde luego, cualquier deficiencia en el presente trabajo es responsabilidad exclusiva del autor.

1 Es necesario explicar la concepción subyacente a esta idea: el "objeto" de una ciencia no le es "pre-existente"; toda ciencia comienza su labor teórica elaborando el concepto de su objeto. 
ción y clases de equivalencia y serán el objeto de una operación de medida económica. El problema inicial que se plantea para la teoría económica es el de la construcción del espacio homogéneo al interior del cual esas relaciones de equivalencia y la operación de medida son inteligibles en tanto expresión objetiva de relaciones sociales. Ese espacio homogéneo no es otro que el espacio de la mercancía o del valor.

La polémica con el pensamiento mercantilista conduce a la teoría económica a la exclusión del dinero del campo analítico inicial y a la necesidad de elaborar los conceptos de precio y de mercancía independientemente de cualquier referencia al dinero. ${ }^{2}$ La teoría económica tomará como punto de partida para llevar a cabo esta tarea, la noción de "objetos físicamente determinados". Así, los valores de uso, bienes, cosas y objetos materiales configuran un elemento esencial de la definición de la mercancía. Esta es concebida como un objeto físicamente determinado al que algún dispositivo social le asocia un coeficiente que constituye su medida económica (i.e., su precio). Tomando en cuenta que la construcción del concepto de mercancía corresponde a la construcción de un espacio homogéneo de medida, espacio que está dotado de ciertas propiedades matemáticas, ¿cuál es la pertinencia de la noción de "objetos físicamente determinados" para la tarea de elaborar el concepto de mercancía?

En este ensayo se presenta una referencia inicial para ubicar este problema en la historia del pensamiento económico (primera parte). Posteriormente, se somete a un análisis crítico la manera en que la teoría del equilibrio general vincula a la noción de objetos físicos y heterogéneos con el concepto de mercancía (segunda parte). Además, se examina la articulación entre la definición de los precios y la anterior concepción de la mercancía (tercera parte). El análisis pretende demostrar la existencia de una debilidad lógica fundamental en toda teoría que tenga el mismo objetivo (la construcción de un concepto de mercancía) y comparta el mismo punto de partida (los objetos físicos y heterogéneos como conjunto de referencia inicial). Por último, en el Apéndice se presentan los elementos de la teoría de la medida, así como de la concepción del intercambio como operación de medida que son importantes desde la perspectiva del presente ensayo.

2 Para un análisis e interpretación de la polémica antimercantilista, con referencia al problema de la exclusión del dinero, véase Nadal (1983). 


\section{DEFINICIÓN DEL PROBLEMA}

Habiéndose expulsado al dinero del campo analítico inicial, la teoría económica se enfrenta a una colección de bienes heterogéneos, inconmensurables entre sí, y que serán objeto de las relaciones económicas. Cada uno de estos objetos está especificado materialmente y en una cantidad determinada en su unidad de medida. Además, las unidades de medida están definidas en las dimensiones adecuadas a las características físicas de cada objeto. En este sentido, la teoría del valor tiene como primer objetivo el restituir la unidad perdida con la exclusión del dinero, estableciendo un espacio homogéneo de medida de los objetos económicos. En otros términos, se trata de la construcción de la dimensión adecuada de los objetos económicos, o sea de las mercancías. Esa dimensión no es otra cosa que el espacio homogéneo del valor, dimensión en la cual las magnitudes numéricas expresan relaciones sociales. La teoría del valor no sólo debe resolver el problema de la inconmensurabilidad de los objetos físicos heterogéneos, sino que debe explicar en qué forma las magnitudes numéricas que resultan de la solución a dicho problema constituyen la expresión de relaciones sociales.

La teoría del valor puede considerarse como una parte de la teoría de la medida y, por lo tanto, es necesario analizarla a la luz de los principios que rigen la medición de cualquier magnitud, examinando si los procedimientos involucrados en la construcción del espacio de la mercancía son correctos. ${ }^{3}$ En particular, se trata de abordar el problema del estatuto teórico de los objetos físicos o "valores de uso" en tanto son considerados como punto de partida del análisis económico posmercantilista. ${ }^{4}$

Marx es probablemente el único autor que percibió la naturaleza del procedimiento analítico que la economía política (y con ella, toda teoría económica) debía seguir para elaborar un concepto de mercancía. Así, independientemente de cualquier apreciación sobre

3 El primer análisis que se sitúa en esta perspectiva es el de Fradin (1973).

4 El procedimiento de tomar como punto de partida a un conjunto de objetos físicos es considerado como una hipótesis por Benetti y Cartelier (1980). Esta hipótesis, llamada de "nomenclatura", desempeña diversas funciones lógicas específicas a cada teoría económica y se presenta con diversas modalidades. Estos autores analizan el papel de esta hipótesis en la economía política clásica y en la teoría neoclásica. En relación a esta última, se concentran en las funciones de la "hipótesis de nomenclatura" en la especificación de los agentes económicos. 
la solución que el propio Marx ofrece, lo cierto es que este autor es el primero en presentar correcta y rigurosamente los términos del problema que nos ocupa. En este contexto, es posible reinterpretar la crítica de Marx a la economía política en el sentido de que el problema inicial no es el de la medida del valor de las mercancías, sino el de las condiciones de posibilidad del intercambio. Los economistas, dice Marx, dan por descontado que el intercambio es una relación de equivalencia, $y$, sobre esta, base buscan medir el valor de cambio. Esto implica que las categorías de "precio" y "mercado" son un dato preexistente del análisis. Es por ello que a los economistas les preocupa el problema de la "buena medida del valor".5 Para Marx, en cambio, lo que interesa inicialmente es establecer las condiciones que permiten concebir el intercambio como relación de equivalencia, tomando en cuenta que el valor, en tanto expresión de relaciones sociales, es enteramente independiente de la materialidad de los objetos físicos. La posición de Marx puede ser apreciada en dos momentos de su crítica a Bailey, quien olvida (según Marx) que si decimos que

$$
x \text { yardas de tela }=y \text { libras de paja }
$$

la igualdad hace de la tela y la paja, en esas cantidades, objetos equivalentes: "No es en tanto paja y tela que estos objetos son iguales, sino en tanto que son equivalentes" (Marx, 1976: 165).

Más adelante, Marx presenta el problema en términos de la necesidad de construir un espacio homogéneo que permita realizar una comparación a través de una medida entre dos objetos diferentes. A la afirmación de Bailey de que el valor, como la distancia, solamente tiene sentido en tanto comparación entre dos objetos, Marx (1976: 169) responde:

Un objeto está a una cierta distancia de otro, la distancia es en efecto una relación entre uno y otro objeto, pero al mismo tiempo la distancia es algo distinto de esta relación entre dos

5 El caso más claro es el de Ricardo, cuya preocupación por encontrar una medida de valor inmune a distorsiones "parásitas" ocupa un papel central en su obra. La concepción de Ricardo (1951) sobre la operación de medida, explicada en la referencia a Destutt de Tracy en el capítulo XX sobre "Valor y Riquezas", es esencialmente correcta. Sin embargo, dicha concepción está acompañada por una confusión sobre la realidad ontológica de la unidad de medida que lo lleva a una incomprensión de las propiedades de la operación de medida. 
cosas. Es una dimensión del espacio, es una longitud que puede expresar también la distancia entre dos objetos además de aquellos que están siendo comparados. (. . .) Si hablamos de la distancia como una relación entre dos objetos, suponemos algo "intrínseco", alguna "propiedad" de los objetos mismos, que les permite estar distantes el uno del otro. ¿Cuál es la distancia entre la letra A y una mesa? Tal pregunta no tendría sentido. Cuando hablamos de la distancia entre dos cosas, hablamos de su diferencia en el espacio. Suponemos que las dos están situadas en el espacio, que ambas son puntos en el espacio. Así, las igualamos como existencias del espacio, y solamente después de haberlas igualado desde el punto de vista del espacio, sub specie spatii, podemos diferenciarlas en tanto puntos diferentes del espacio. El hecho de pertenecer al espacio constituye su unidad. (Traducción nuestra.)

De esta manera, la teoría económica debe proceder inicialmente a la construcción del espacio homogéneo de la mercancía que permita discurrir sobre las relaciones de intercambio en las que intervienen objetos heterogéneos: el hecho de pertenecer a ese espacio homogéneo permite considerarlos como mercancías. El intercambio sería entonces la operación que permite clasificar a los objetos económicos en clases de equivalencia, asignándoles un coeficiente numérico que constituye su medida de valor. Es por esto que el problema que la teoría económica debe resolver inicialmente no es el de la "medida del valor" sino el del "valor como medida" de los bienes en el intercambio (es decir, en tanto mercancías).

Lo que destaca en los planteamientos de Marx es el principio general de toda teoría del valor en tanto teoría de la medida: la operación de medida requiere de la reducción de los objetos medidos a magnitudes conmensurables, homogéneas. Se ha demostrado que es posible establecer un vínculo entre la relación de intercambio concebida como relación de equivalencia y la operación de medida real. ${ }^{6}$ En este contexto, el intercambio como relación de equivalencia es la operación a través de la cual a una magnitud económica (i.e., a una cantidad de la mercancía) se le asocia un elemento del espacio de medida (i.e., un valor). En este procedimiento interviene una operación de abstracción matemática en la que las mercancías (que en apariencia pueden ser distinguidas desde el punto de vista de sus propiedades físicas) son reducidas a cantidades o magnitudes homo-

6 Fradin (1973) y Nivollet (1980). Sobre este punto, véase el Apéndice. 
géneas antes de poder ser consideradas como siendo iguales desde el punto de vista del valor.

Ahora bien, la operación de medida de que se trata es una operación económica, $y$, como tal, debe expresar relaciones sociales. Es decir, el coeficiente numérico que es la medida de la mercancía constituye la expresión de relaciones sociales y no de atributos físicamente determinados de los objetos (peso, longitud, etc.). Tomando en cuenta los elementos de la teoría de la medida presentados en el Apéndice, se concluye que la tarea inicial de la teoría económica consiste en construir el espacio homogéneo de medida del valor, al interior del cual las relaciones de equivalencia del intercambio son inteligibles como expresión de relaciones sociales. Esta primera tarea no es otra cosa que la elaboración del concepto de mercancía. Sin este concepto, los enunciados ulteriores de la teoría sobre la determinación de precios y distribución del ingreso serán ininteligibles. En este contexto, la teoría debe explicar el procedimiento de construcción del conjunto de magnitudes y del espacio de medidas, así como enumerar $y$ justificar sus propiedades elementales.

\section{LAS MERCANCÍAS COMO OBJETOS FíSICOS}

En esta sección se examina el procedimiento seguido por la teoría del equilibrio general en la construcción del concepto de mercancía. El análisis estará centrado sobre la obra de G. Debreu (1959), no sólo porque pretende explicar a través de un análisis axiomático la forma en que se construye el concepto de mercancía, sino porque se trata de la obra de uno de los autores más representativos de dicha corriente y cuyos teoremas constituyen la base de la teoría contemporánea del equilibrio general. Como ya se indicó, la pregunta central se refiere a la naturaleza de las magnitudes que son el objeto de la operación de medida económica (o sea, las mercancías). Lo que interesa es desentrañar si es adecuado considerar que esas magnitudes son precisamente los objetos físicos, o "valores de uso", que configuran el punto de partida del análisis real posmercantilista.

Debreu inicia su análisis afirmando que los conceptos de mercancía y de precio son conceptos "duales", aunque el sentido preciso de la dualidad no está explicado. Señalando que a toda mercancía le es asociado un precio, Debreu parece implicar que la noción de mercancía es inteligible antes e independientemente de que se le asocie un 
precio. En efecto, para este autor, la mercancía es un objeto físico bien determinado:

...en resumen, una mercancía es un bien o servicio completamente especificado física, temporal y espacialmente (p. 32). (Traducción nuestra.) finito.

Además, el análisis considera al conjunto de mercancías como

Para este autor, los parámetros para especificar cada mercancía son de tres tipos: propiedades físicas, fecha de disponibilidad y localización. En el caso de los últimos dos, los intervalos y las regiones se expresan en unidades "elementales": de la misma duración para los primeros, y de tal forma que los puntos al interior de una región sean indiferenciables desde el punto de vista del análisis. En el caso de las propiedades físicas, aunque no se explican los parámetros para definirlas, es suficiente con introducir el supuesto de que los agentes económicos pueden distinguirlas. Pero en lo que concierne a las cantidades físicas de todas las mercancías, Debreu introduce un elemento fundamental: dichas cantidades pueden ser expresadas por cualquier número real. En otras palabras, cualquiera que sea el conjunto de propiedades físicas que caracterizan a un bien específico, el número de unidades puede ser expresado incluso por un número irracional. ${ }^{7}$

Debreu está introduciendo de esta manera el postulado crucial de la divisibilidad perfecta de todos los bienes. En efecto, la expresión de las cantidades físicas de cualquier bien a través de números reales implica que es posible llevar tan lejos como se quiera la división de cualquier bien y siempre tener un objeto que responda a las mismas propiedades físicas del objeto inicial. ${ }^{8}$ La obra de Debreu pre-

7 Es importante no perder de vista que se trata de números reales que son inteligibles únicamente después de especificarse la unidad de medida o la dimensión adecuada a las propiedades físicas de cada mercancía. Se trata, pues, de números reales con dimensión y no de números puros.

$8 \mathrm{La}$ utilización de números reales está explícitamente indicada para las cantidades de los objetos físicos. Las fechas y lugares se expresan en intervalos y regiones "elementales", dándose a entender que los subíndices que serían utilizados para denotar fecha y localización de disponibilidad de una mercancía serán números naturales. (Los conjuntos de intervalos y regiones pueden ser finitos y numerables). Así, el número de mercancías parece seguir siendo un número finito. Sin embargo, Debreu señala que si las fechas fueran infinitas el número de mercancías también lo sería (nota 2 del capítulo 2). Esto quiere decir que el número de fechas incide per se sobre el número de mercancías. 
senta una justificación, pero no una explicación teórica, para la introducción de este postulado. Sobre la justificación encontramos un enunciado sorprendente en el siguiente pasaje:

Una cantidad de camiones bien definidos es un entero; pero se supondrá, en cambio, que esta cantidad puede ser un número real. Este supuesto de divisibilidad perfecta se impone por el estado actual del desarrollo de la economía; es perfectamente aceptable para un agente económico que consume o produce una gran cantidad de camiones (p. 30).

Aquí encontramos una ambigüedad importante. La justificación para poder hablar de "3.141592 tropeladoras" o de $\sqrt{2}$ hornos Bessemer" proviene de la aceptación de los agentes económicos; pero cabe preguntar en qué momento del proceso de formación de precios la representación de cantidades físicas por números reales es aceptable desde el punto de vista contable para los agentes. Es evidente que se tiene que evitar considerar como mercancías a aquéllas que solamente existen en los cálculos subjetivos de los agentes, pues en estricto rigor no se puede hablar de la existencia económica de dichos objetos. El enunciado sobre la aceptabilidad (contable) de dicho postulado de divisibilidad perfecta por los agentes sólo es inteligible cada vez que se ha anunciado un vector de precios y se han anunciado las transacciones que, con ese sistema de precios, los agentes están dispuestos a llevar a cabo. La única noción de mercancías virtuales inteligible para la teoría es la de aquellas mercancías que tienen una existencia económica (por ejemplo, son el objeto de un contrato que prevé su entrega en una fecha futura), es decir que son socialmente reconocidas aunque no existen físicamente. La "aceptación" por los agentes no debe ser interpretada como equivalente a incluir a las "mercancías subjetivas" en el espacio de las mercancías: éste está reservado exclusivamente a los objetos socialmente significativos.

Es posible seguir ahora con el análisis de las características del conjunto de mercancías. Debreu propone lo siguiente:

Se supone que sólo existe un número finito $\ell$ de mercancías diferentes (distinguishable commodities); se indican por un índice $h$ que va de 1 hasta $\ell$. También se supone que la cantidad de cualquiera de ellas puede ser un número real.

Pero, como se verá, este enunciado introduce serias ambigüedades por lo que toca a las mercancías "virtuales" que integran el conjunto de mercancías. 
Sin embargo, nuestro autor señala que es posible generalizar el alcance de la teoría al caso de un conjunto con un número infinito de mercancías (al referirse a un número infinito de fechas) y que muchos de los resultados de la teoría se mantienen. A reserva de llevar a cabo una investigación sobre la validez de esta afirmación (y, en particular, sobre las condiciones más restrictivas que deben ser incorporadas y los resultados más débiles que pueden ser alcanzados) ${ }^{9}$ el aspecto más importante es el de la compatibilidad entre el supuesto de un número infinito de mercancías y las bases mismas de la teoría económica. En particular, faltaría explicar el sentido de dicho supuesto en el contexto de una teoría económica que de alguna manera introduce una noción de escasez (subyacente en la noción de dotación inicial) y supuestos de no saciedad.

Hasta aquí se han examinado algunas de las ambigüedades relacionadas con la justificación del postulado de perfecta divisibilidad a través de su "aceptación" por los agentes económicos. Ahora bien, es importante observar que Debreu no explica el porqué dicho postulado es aceptable para la teoria, que sería lo indicado en el ámbito de un análisis axiomático.

En efecto, desde el punto de vista de la teoría el problema se plantea en términos de explicar la introducción de un postulado que no sólo es muy fuerte, sino que es radicalmente contrario a la idea inicialmente propuesta sobre las mercancías como objetos físicamente determinados. Debreu termina por descartar la "justificación" de que los agentes encuentran dicho postulado aceptable como "irrelevante para el desarrollo lógico de la teoria" (p. 35). En efecto este autor procede de la manera siguiente: pretendiendo llevar a cabo un análisis axiomático, Debreu se permite "introducir el concepto de mercancía por medio de ejemplos" (p. 29), para posteriormente aceptar que dichos ejemplos son irrelevantes para la construcción teórica del espacio de mercancías, mismo que simple y llanamente es presentado como igual a $R^{\ell}$ "en el lenguaje de la teoría". Este último enunciado constituye un postulado y no el resultado de un procedimiento riguroso de construcción de un concepto. Precisamente, en el lenguaje de la teoría, no se nos dice nada sobre por qué es posible concebir que "3.1459" o " $\sqrt{2}$ " sigue siendo cantidades de camiones, máquinas herramienta, espectáculos de teatro, servicios de peluquería, etc. A continuación se explica la razón por la cual Debreu debe mantener 
abierta la posibilidad de que las cantidades de las mercancías distinguibles físicamente sean números reales.

El intercambio puede ser concebido como una operación de medida a través de la cual es posible asociar una medida a las magnitudes económicas; esa medida es un coeficiente numérico por el cual hay que multiplicar la unidad de medida para formar la magnitud considerada. Desde este punto de vista, el intercambio es una operación de medida económica distinta de toda operación de medida física. Debreu señala explícitamente que a cada mercancía le está asociado un número real que es su precio (p. 32); así la operación de medida económica puede ser considerada como una función $v$ cuyo dominio es el espacio de magnitudes $X$ y cuyo contradominio es el espacio de los reales (positivos unión con el $O$ ):

$$
v: X \longrightarrow R^{+} \cup\{O\} \text {. }
$$

En otros términos, el espacio de medidas $M$ es isomorfo con respecto a los reales. De esta manera la operación de medición puede desembocar en un número irracional: supongamos que tenemos el valor de $x$ unidades físicas del bien $i$ :

$$
v\left(x_{i}\right)=r,
$$

entonces $r$ es la medida de $x_{i}$. En este sentido, o sea $\alpha$ la medida de una unidad física del bien $i$,

$$
v\left(u_{i}\right)=\alpha,
$$

y tenemos la posibilidad de escribir

$$
x \cdot v\left(u_{i}\right)=r
$$

en donde $x$ ya no es más que un número puro, sin dimensión. Y por la definición del espacio de medida, $\alpha, r \in R^{+} \cup\{O\}$. Pero en este caso, como estamos frente a un producto externo, también tenemos que mantener abierta la posibilidad de que $x$ sea un número real. ${ }^{10}$

En efecto, si aceptamos que tanto $r$ como $\alpha$ sean números reales,

10 Tanto en el caso $v\left(x_{i}\right)=r$, como en $v\left(u_{i}\right)=\alpha$, estamos en presencia de un producto externo, con todas sus propiedades. Conviene señalar que las $\alpha$ sólo están definidas en términos del valor de una unidad física de cada bien $i$. Pero $\alpha$ no necesariamente corresponde a una unidad de valor. 
tenemos las siguientes posibilidades: pueden pertenecer a $N, Q \circ I$. Para mantener abiertas todas las posibilidades, tenemos que imponer ciertas propiedades para los elementos $x_{i}$ del conjunto de magnitudes. Así, como $r$ puede ser un número natural y $\alpha$ un irracional, necesariamente se tiene que mantener abierta la posibilidad de que $x$ sea igualmente un irracional porque $I$ es el único conjunto que no es cerrado bajo la multiplicación. De esta manera sería posible mantener $x \cdot \dot{\alpha}=r, \operatorname{con} r \in N$ y $\alpha \in I$.

En otros términos, si exigimos que el espacio de medida sea isomorfo con respecto a los reales, entonces las cantidades (físicas) de los bienes deben ser también números reales. De este modo, el que las cantidades de los bienes físicamente determinados sean números reales se nos impone por las características de la operación de medida y no "por el estado actual de desarrollo de la economía" (Debreu). Se trata de dos razones enteramente distintas en cuanto a su naturaleza; si este autor posteriormente considera a la segunda como irrelevante para la construcción de la teoría, en ningún momento llega a explicar cuál es el procedimiento seguido en la construcción del espacio de mercancías. Esta laguna se agrava con la insistencia en identificar mercancías con "bienes física, temporal y espacialmente especificados", con lo cual se mantiene una profunda ambigüedad en lo que concierne a la naturaleza del concepto.

Habiéndose explicado la razón por la cual es indispensable aceptar que las cantidades de las magnitudes sean expresadas por números reales, es preciso profundizar sobre las implicaciones de lo anterior examinando las características del conjunto de mercancías. Aparentemente el conjunto de mercancías está formado por una colección finita de mercancías. Sea $E$ dicho conjunto, con un número $\ell$ (finito) de mercancías diferentes.

$$
E=\{1,2, \ldots, \ell\}
$$

en donde cada elemento está especificado de acuerdo a sus propiedades físicas, fecha y lugar de disponibilidad: éste sería el conjunto de las $\ell$ distinguishable commodities. Si se dice que la cantidad de cada uno de estos bienes puede ser un número real, tenemos entonces un segundo conjunto

$$
\bar{E}=\left\{x_{1}, x_{2}, \ldots, x_{\ell}\right\}
$$


en donde cada $x_{i}$ es un número real y representa una cantidad de unidades del bien $i$. El conjunto $\bar{E}$ puede ser interpretado de dos maneras distintas:

- o bien cada $x_{i}$ forma un solo elemento del conjunto (por ejemplo, "7 camiones" forma un solo elemento y no 7);

- o bien cada $x_{i}$ representa otros tantos elementos del conjunto (los "7 camiones" cuentan como 7 elementos).

En el primer caso, tendríamos un conjunto $\bar{E}^{\prime}=\left\{X_{1}, X_{2}, \ldots\right.$, $X_{\ell}$ en el que cada $X_{i}$ representa una cierta cantidad de unidades del bien $i$ que son consideradas como, o mejor aún, que constituyen un solo elemento; en este caso, card $\bar{E}^{\prime}=\ell$. Pero aquí no hay desagregación posible y surgen problemas para concebir las dotaciones iniciales, así como la realización de las transacciones. En consecuencia, es evidente que el conjunto de mercancías no puede ser $\bar{E}^{\prime}$ porque éste es incompatible con el desarrollo ulterior de la teoría. En el segundo caso, tenemos un conjunto en el que las cantidades de los bienes, expresadas en números reales, son infinitamente divisibles y cada unidad de esas cantidades constituye un elemento del conjunto. Sea $\hat{E}$ dicho conjunto:

$$
\hat{E}=\bigcup_{i=1}^{\ell} U_{i}
$$

en donde los elementos de $\hat{E}$ no son las $U_{i}$ sino los elementos de las $U_{i}$, y en donde

$$
U_{i}=\left\{x \mid x=\alpha \cdot u_{i}, \alpha \in R^{+}\right\}
$$

(para cada mercancía, $u_{i}$ denota la unidad definida de acuerdo a sus propiedades físicas). De esta manera, el conjunto $\hat{E}$ está compuesto por una infinidad de elementos pues cada una de las "mercancías diferentes" es infinitamente divisible; así para toda $U_{i}$, cada una de las unidades que la integran constituye una unidad del bien $i$. Como se trata de números reales, existe una infinidad de unidades de cada mercancía diferente. Este es el verdadero significado dél hecho de que $x$ pueda ser un número real. Es importante hacer hincapié sobre el significado del postulado sobre la expresión de las cantidades de bienes físicos en o a través de números reales. La operación de divi- 
sión se puede llevar tan lejos como se desee, pero siempre estaremos frente a objetos físicos de la misma naturaleza y con las mismas propiedades; y como el postulado implica divisibilidad perfecta al infinito, estamos en presencia de un número infinito de mercancías. Por otra parte, en vista de que ningún objeto físico tiene la propiedad requerida de divisibilidad perfecta, se demuestra la imposibilidad lógica de utilizar números reales para expresar cantidades de objetos físicamente especificados. ${ }^{11}$

\section{OBJETOS FísICOS Y PRECIOS}

Partiendo del supuesto inicial de la existencia de $\ell$ mercancías físicamente diferenciables, el problema de la homogeneización de la medida de dichos objetos necesariamente hace su aparición en la obra de Debreu. Así, se plantea una disyuntiva crucial sobre las características del espacio de mercancías, definido simplemente como $R \ell$ :

- o bien $R^{\ell}$ se compone de $\ell$ dimensiones heterogéneas, que son precisamente las que permiten la distinción de las $\ell$ mercancías físicamente especificadas;

- o bien $R^{\ell}$ se compone de $\ell$ dimensiones que son homogéneas y se necesita redefinir la manera en que se presenta el supuesto sobre las $\ell$ mercancías diferenciables.

La presencia de las $\ell$ dimensiones heterogéneas es sin duda la interpretación conforme a la determinación de las mercancías por sus propiedades físicas. Cada agente puede ubicar correctamente en $R^{\ell}$ a todas las mercancías a través de sus propiedades físicas y por tanto puede siempre asociar correctamente a cada mercancía el precio correspondiente. La otra posibilidad implica que de una manera u otra se ha llevado a cabo una homogeneización de las dimensiones o unidades de medida, y que se puede hacer abstracción de la diferenciación física que inicialmente permitía especificar a las mercancías. Será necesario, en este caso, introducir elementos adicionales que permitan a los agentes ubicar correctamente en $R^{\ell}$ a las mercancías, permitiendo así la asociación adecuada de precios y mercancías.

11 Sobre las propiedades del conjunto de los números reales, véase el Apéndice. 
Para analizar estas dos posibilidades, debemos tomar en consideración la articulación entre el espacio de mercancías y el espacio de precios. Según Debreu, a cada mercancía le está asociado un número real, su precio. Es posible entonces definir "el valor de una acción 'a' relativamente a un sistema de precios $p$ como $\sum_{h=1}^{\ell} p_{h} \cdot a_{h}$ i.e., el producto interno $p \circ a$ " (p. 33). Aparentemente estamos en presencia de una homogeneización a través de los precios. Sin embargo, en la peor tradición nominalista, Debreu ofrece una posible interpretación de la noción de precio en este contexto: "el precio $\left(p_{h}\right)$ puede ser interpretado como el monto pagado ahora por un agente por cada unidad de la mercancía $h$ que se le hará disponible" (p. 32). Debreu se limita posteriormente a definir un sistema de precios como un punto de $R^{\ell}$, y $\sin$ información adicional quedan dos interpretaciones posibles:

- $p$ es un punto de $R^{\ell}$ : sus componentes son números puros;

- $p$ es un punto de $R^{\ell}$ : sus componentes son números reales con unidades (es decir, dimensionados).

En el primer caso, se tiene que excluir la posibilidad de concebir al vector $a$ como integrado por $\ell$ componentes heterogéneos, porque en esa eventualidad el producto escalar $p \cdot a$ no es concebible. En el enunciado teórico correspondiente, Debreu se limita a señalar que una acción $a$ de un agente es "un punto de $R^{\ell}$, el espacio de mercancías" (p. 35), con lo cual se aseguran las condiciones que permiten realizar el producto escalar mencionado. ${ }^{12}$ Sin embargo, esto significa que Debreu está simplemente postulando la homogeneidad de las dimensiones para las $\ell$ mercancías; dicho procedimiento hace posible el producto escalar $p \cdot a$, pero contradice el supuesto inicial haciendo abstracción de la especificación de las mercancías a través de sus propiedades físicas.

En este caso, el problema es el de mantener el supuesto de que existen $\ell$ mercancías distinguibles. Este supuesto es fundamental para la inteligibilidad del proceso de mercado pues es lo que permite a los agentes asociar correctamente precios (en su función paramétrica) y mercancías: así pueden calcular correctamente el valor de

12 El problema de las condiciones requeridas para poder calcular el producto escalar $p \cdot a$ fue identificado originalmente por Fradin (1973). 
cualquier acción, sus restricciones de presupuesto o la función objetivo. ${ }^{13}$ Sin la posibilidad de que todos los agentes asocien de la misma manera precios y mercancías, el valor de la misma acción sería distinto para cada agente, introduciéndose una confusión análoga a la que reinaría si se tuviese multiplicidad de precios.

Ahora bien, para mantener a salvo el supuesto de la distinción entre las $\ell$ mercancías sin referencia aparente a las propiedades físicas es suficiente aceptar que la posición de cada componente en los vectores $a$ (puntos de $R^{\ell}$ ) puede informar a los agentes sobre el tipo de bien de que se trata, permitiendo la asociación correcta con su precio. Se necesitaría introducir la condición siguiente: los agentes deben adoptar un orden común en sus listas de bienes de tal manera que los mismos componentes (desde el punto de vista de su posición en el vector $a$ ) se refieran al mismo tipo de mercancías. En los términos de Debreu, las mercancias ya no serian bienes fisicamente especificados sino bienes especificados por su posición en el interior de cada vector $a$. Así, bastaría con señalar que los agentes adoptan antes e independientemente del proceso de mercado el orden establecido por el conjunto

$$
E=\{1, \ldots, \ell\}
$$

que atribuye una posición a cada mercancía diferenciable. ${ }^{14}$ Los agentes podrían en todo momento discriminar entre acciones, calculando el valor correcto de cada una.

Sin embargo, la referencia a las propiedades físicas como caracterización de las mercancías no se abandona sino en apariencia. El problema de la utilización de números irracionales para las cantidades de los bienes sigue en pie, pues, si bien el orden en el vector $a$ es lo que permite distinguir una mercancía de otra, éstas siguen siendo los bienes y servicios físicamente especificados. Así, los agentes saben que la posición de un componente de $a$ permite identificar objetos físicos: éstos no han desaparecido y solamente estamos en presencia de una seudohomogenización. En efecto, la seudohomogenización

13 La función del supuesto que permite la asociación "correcta" de precios a mercancías es lo que Benetti y Cartelier (1980) llaman la función de "objetividad social" de la hipótesis de nomenclatura.

14 Cada agente respetaría el orden del mismo set of labels, como llaman Koopmans y Bausch (1959) al conjunto $E$. 
consiste en substituir una manera de distinguir (entre mercancías) por otra, sin que se elimine la referencia a los objetos físicos. La prueba es que en este caso, que parece ser el camino seguido por Debreu, no existe información sobre el sentido preciso de la dimensión homogénea que caracteriza al espacio de las mercancías; parecería que la seudohomogenización corresponde a una simple abolición de la heterogeneidad de las $\ell$ dimensiones iniciales. En este caso, el producto escalar $p \cdot a$ sí es posible, pero es perfectamente ininteligible desde el punto de vista de la teoría económica. ${ }^{15}$

Es necesario examinar la otra manera de introducir las condiciones que permiten el mencionado producto escalar considerando al vector $a$ como integrado por $\ell$ componentes heterogéneos. Si el vector de precios se define como un punto de $R^{\ell}$ cuyos componentes son números con unidades, es decir precios relativos, y escogiendo un numéraire, el producto escalar $\boldsymbol{p} \cdot \boldsymbol{a}$ permite expresar el valor de una acción como una suma de cantidades homogéneas. Para evitar el problema de la indeterminación de los precios relativos cuando el $n u$ méraire resulta ser un bien libre, se escoge un bien compuesto. Sea el vector $N$ dicho bien,

$$
N=\left(u_{1}, u_{2}, \ldots, u_{\ell}\right)
$$

integrado por una unidad de cada una de las $\ell$ mercancías. El vector de precios se escribe como un vector de precios relativos:

$$
p=\left(\frac{\alpha_{1} N}{u_{1}}, \ldots, \frac{\alpha_{\ell} N}{u_{\ell}}\right)
$$

en donde cada componente expresa la fracción del bien compuesto $N$ que se intercambia por unidad de cada mercancía. Por definición,

15 Existiendo una seudohomogenización en la obra de Debreu, el enunciado adecuado para definir a las mercancías debería ser el siguiente: las mercancías son en un número $\ell$; se distinguen por la posición que ocupan en el vector $a$, cuyo orden es aceptado uniformemente por todos los agentes; el número $x_{i} \in R$ es expresión del número de unidades de la mercancía que ocupa la posición $i$ en el vector $a$. La referencia a las mercancías como bienes físicos, y los problemas que ella conlleva, no desaparecen. (De hecho, para poder ubicar correctamente a los bienes en su posición adecuada, los agentes deben referirse a las propiedades físicas de los bienes.) 


$$
p \cdot N=\left[\frac{\alpha_{1} N}{u_{1}} u_{1}+\frac{\alpha_{2} N}{u_{2}} u_{2}+\ldots+\frac{\alpha_{\ell} N}{u_{\ell}} u_{\ell}\right]
$$

y como por definición el precio del numéraire debe ser $p \cdot N=1$, tenemos la siguiente condición para los coeficientes $\alpha_{i}$ :

$$
\Sigma \alpha_{i}=1
$$

En realidad, estos coeficientes no son otra cosa que los precios relativos de los bienes expresados en el numéraire. Por lo tanto, estamos en presencia de la condición definida por Debreu (p. 81) según la cual la búsqueda del equilibrio se restringe a los vectores de precio que han sido normalizados (intersecciones del ortante positivo y el conjunto de puntos que verifican la ecuación $\Sigma p_{i}=1$ ). Ahora bien, el proceso de normalización parte de que la correspondencia de demanda neta total $\zeta$ es homogénea de grado cero en los precios y definiendo $k=1 / \sum_{i=1}^{\ell} p_{i}$ tenemos

$$
\zeta\left(\frac{1}{\sum_{i=1}^{\ell}} p_{i}\right) p=\zeta(p)
$$

La condición de normalización es entonces la misma que permite la definición del numéraire. El problema que surge es el de la inteligibilidad de dicha condición de normalización, tomando en cuenta que las $p_{i}$ son coeficientes o tasas de substitución física de los $\ell$ bienes heterogéneos y el numeraire. Enfatizando la expresión:

$$
\Sigma p_{i}=\left(\frac{\alpha_{1} N}{\left(u_{1}\right)}+\ldots+\frac{\alpha_{\ell} N}{\left(u_{\ell}\right)}\right)
$$

cada uno de los precios relativos expresa un coeficiente dotado de una dimensión compuesta: la fracción $\alpha_{i}$ de $N$ por unidad del bien $i$. En general, con el supuesto de $\ell$ mercancías (físicamente) diferen- 
ciables, dichas dimensiones compuestas serán igualmente heterogéneas y la suma no puede ser llevada a cabo. Los coeficientes $p_{i}$ no pueden ser considerados como números puros sin dimensión; el procedimiento para definirlos no permite anular las unidades en las que se expresan las cantidades (en contra de la concepción de Jevons y Walras). En estas circunstancias, llegamos a un resultado interesante: el numéraire $N$ puede ser construido cuando la expresión $\Sigma p_{i}$ tiene un sentido preciso, es decir cuando los elementos $p_{i}$ pueden ser sumados; pero en ese caso, los precios tienen la misma dimensión (compuesta), y por lo tanto es redundante construir un numéraire porque las dimensiones de las $\ell$ mercancías distinguibles ya han sido previamente homogenizadas. La condición de normalización sólo es inteligible cuando ya existe una homogenización; en conclusión, la construcción del numéraire requiere de la supresión del supuesto que lo hace necesario.

Por otra parte, la introducción de un numéraire no puede considerarse como elemento suficiente para la determinación del concepto de mercancía. La prueba es que aún haciendo abstracción de las dificultades para definir un numéraire en la obra de Debreu, se mantienen los problemas señalados anteriormente: los precios relativos (tasas de substitución física) siguen siendo números reales, al igual que las cantidades de los bienes; por lo tanto, las mercancías no pueden ser objetos físicamente especificados. En última instancia, la relación entre el numéraire y las mercancías sólo es inteligible cuando se ha construido el espacio de magnitudes homogéneas. ${ }^{16}$

\section{OBSERVACIONES FINALES}

Si regresamos ahora al proceso de homogenización implícito en la operación de abstracción introducido por la relación de equivalencia, vemos que es en tanto mercancías que los objetos físicos están siendo medidos; o sea, es en tanto mercancías que se les asigna un precio en el intercambio. Pero, en tanto mercancías los objetos constituyen magnitudes homogéneas ( $y$ dejan de existir para la teoría en tanto objetos físicos). ¿Cómo se constituyen en magnitudes homogéneas? Precisamente es la operación de medida (en este caso a través

16 Cabe señalar que lo anterior es independiente de otros problemas fundamentales que plantea la introducción del numéraire (por ejemplo, su vinculación con el postulado de la unicidad de precios, etc.). 
del intercambio) lo que debería permitir la definición específica de la magnitud o dimensión en cuestión. ${ }^{17}$ En el caso considerado aquí el conjunto de magnitudes (que son objeto de la operación de medida) es denotado como $E^{*}$ : éste ya no está compuesto por bienes físicos heterogéneos, sino por mercancías (i.e., magnitudes homogéneas). Los elementos de $E^{*}$ deben ser inteligibles económicamente, es decir, en la dimensión social del valor. El conjunto de valores o el espacio de medida es $M$, cuyos elementos son las clases de equivalencia en las que el intercambio coloca o clasifica a las mercancías; de esta manera tenemos que $M=E^{*} / L$. El intercambio es la operación de medida que permite asignar a cada fracción de la mercancía un coeficiente $\alpha \in M$.

Por otra parte, un elemento adicional que permite afirmar que los elementos de $E^{*}$ son magnitudes homogéneas y no bienes físicos heterogéneos es el hecho de que tanto $v\left(x_{i}\right)=r$, como $x \cdot v\left(u_{i}\right)=r$ constituyen productos externos. Es decir, un número $x$ multiplica a una magnitud para llegar a otra magnitud de la misma especie. El producto externo tiene sentido cuando se define sobre un conjunto de magnitudes homogéneas; en este caso es inteligible sólo si se define sobre $E^{*}$ y no sobre $\hat{E}$ que está integrado por elementos heterogéneos.

Además, al definir el espacio de medidas se introduce implícitamente el postulado de que es posible sumar medidas (en este caso valores). Esto introduce ipso facto la posibilidad de "concatenar" o de "agregar" las magnitudes. En otros términos, sea $\alpha$ la medida de $x_{i}, \beta$ la medida de $x_{j}$ y $\gamma=\alpha+\beta$. Esto implica que $\gamma$ es la medida de la magnitud que resulta de la concatenación de $x_{i}$ y $x_{j}$. Esto corresponde a una de las propiedades del producto externo pues

$$
\gamma=v\left(x_{i}+x_{j}\right) \Longrightarrow \gamma=v\left(x_{i}\right)+v\left(x_{j}\right)
$$

en donde $\oplus$ denota la operación de concatenación. Cualquiera que sea el sentido de la operación de concatenación, esto implica que $x_{i}$ y $x_{j}$ son magnitudes homogéneas o deben serlo para que dicha operación sea inteligible. Es evidente que la operación de concatenación en el contex to de la teoría económica sólo tiene sentido en tanto las magnitudes sean mercancías y no bienes físicos heterogéneos, pues la

17 De la misma manera que las operaciones de medida de longitudes, superficies, volúmenes, pesos, etc., permiten definir el sentido numérico de las magnitudes correspondientes. Sobre este punto véase Lebesgue (1975). 
concatenación de una refinería y de $x$ arrobas de trigo no tiene sentido si no se define la dimensión o unidad de medida correspondiente.

Las propiedades que se piden para el espacio de medidas $M(M=$ $E^{*}(L)$ obligan a que éste sea isomorfo a los reales; entonces tenemos que card $E^{*} / L=c$ (donde $c$ es la cardinalidad de $R$ ). Pero esto demuestra que la cardinalidad del conjunto de magnitudes $E^{*}$ no puede ser $\ell$; por lo menos tenemos que card $E^{*}=c$. En consecuencia, el conjunto de partida no puede ser un conjunto de bienes físicos en cantidad finita.

El problema de la infinidad de clases de equivalencia y su articulación con la cardinalidad del conjunto de mercancías se presenta de la siguiente manera. ${ }^{18}$ Dejando de lado por el momento el tema de las magnitudes homogéneas, elementos de $E^{*}$, podemos examinar las implicaciones de la introducción de un postulado de divisibilidad perfecta de los bienes físicos. Sean las cantidades $x_{i}$ y $x_{i}$ de los bienes $i$ y $j$, ambos elementos de $\hat{E}$; la divisibilidad perfecta de esos bienes equivale a considerar que las cantidades $\alpha x_{i}$ y $\alpha x_{j}$ siguen siendo cantidades de esos mismos bienes, con $\alpha \epsilon[0,1] \subset R$. Existiendo tantos puntos en el intervalo $[0,1]$ como en el conjunto $R$, tenemos un número infinito de clases de equivalencia posibles:

$$
\begin{aligned}
& x_{i} L x_{j} \Longrightarrow x_{i}, x_{j} \in C_{i} \\
& \alpha x_{i} L \alpha x_{j} \Longrightarrow \alpha x_{i}, \alpha x_{j} \in C_{\alpha i}
\end{aligned}
$$

En consecuencia, existe por construcción del conjunto $\hat{E}$ y del espacio de medidas $M$ (isomorfo a los reales) una operación suprayectiva e inyectiva. Es suprayectiva porque todo elemento $\alpha$ de $M$ es la imagen de un elemento $x_{i} \in \hat{E}$; inyectiva porque a dos elementos distintos del conjunto $E$ corresponden imágenes distintas. Por lo tanto, la operación de medida es biyectiva y permite asociar a cada elemento de $\hat{E}$ un elemento $\alpha \in M$ de tal manera que toda $\alpha$ es la imagen de un elemento de $E$. En este caso, sabemos que ambos conjuntos deben ser equipotentes, pero como $M$ es isomorfo a los reales,

18 El tema de la articulación de la cardinalidad de ambos conjuntos es abordado por Fradin (1973) y Nivollet (1980). Por otra parte, es necesario explicar cómo el razonamiento en términos de clases de equivalencia implica necesariamente un proceso de homogenización de las magnitudes; igualmente, es importante demostrar que existe (por construcción) una operación suprayectiva e inyectiva entre el conjunto de magnitudes y el espacio de medidas. 


$$
\text { card } M=\text { card } R^{+} \cup\{0\} \text {, }
$$

y esto implica que por lo menos

$$
\text { card } \hat{E}=\text { card } R^{+} \cup\{0\} \text {. }
$$

En otros términos, la estructura y propiedades del conjunto $\hat{E}$ no son independientes de las de $M$, existiendo un isoformismo entre ambos conjuntos.

Pero entonces es crucial determinar la naturaleza de esos elementos que integran el conjunto de partida y que son divisibles al infinito. En el análisis de Debreu, el conjunto inicial no puede ser $\bar{E}$ porque se cae en una contradicción entre la cardinalidad del conjunto de medidas y la de $\bar{E}$. Tampoco puede ser $\hat{E}$ porque en este conjunto se mantiene la heterogenidad de los bienes físicos (el espacio de mercancías propuesto por Debreu en un espacio vectorial de $\ell$ dimensiones heterogéneas). En consecuencia, el conjunto inicial debe ser $E^{*}$, que contiene un número infinito de elementos (o que es equipotente a $R^{+} \cup\{0\}$ ), todos ellos magnitudes homogéneas por la aplicación de una relación de equivalencia: se trata de las magnitudes específicas de la operación de medida que constituye el intercambio. Asi, no nada más la divisibilidad perfecta, sino la homogenización de las magnitudes son condiciones que se imponen por los requerimientos de la operación de medida real. Estas propiedades no corresponden a bienes físicos tangibles: entonces éstos no son las magnitudes objeto de la medida a través del intercambio. ${ }^{19}$

En consecuencia, si se desea tener acceso al espacio de los números reales para discurrir sobre las magnitudes económicas, el conjunto de mercancías no puede ser un conjunto de bienes físicamente especificados. $^{20} \mathrm{El}$ análisis que propone Debreu no contiene un solo

$19 \mathrm{El}$ análisis de Debreu parece indicar que existen mercancías divisibles y no divisibles (véase por ejemplo, nota 3 del Cap. II). Las incoherencias se multiplican: como ya se señaló no existen objetos físicos divisibles indefinidamente; por otra parte, toda mercancía, en tanto magnitud homogénea, es divisible a voluntad, independientemente de sus características en tanto valor de uso. El error de Debreu demuestra que carece de un concepto de mercancía.

20 Esta es una observación válida para cualquier teoría económica en la que el punto de partida sea la asimilación de los objetos físicos (heterogéneos) a las mercancías. Sobre la manera en que el problema de la heterogenidad de dimensiones se presenta en la obra de Sraffa, véase el análisis de C. Salas y A. Valle (1981). Conviene señalar que el sistema de Sraffa también trabaja con 
elemento de información sobre la manera en que se desarrolla lógicamente la teoría para desembocar en la idea de que el espacio de mercancías es $R^{\ell}$ : éste es un postulado y no el resultado de un procedimiento lógico de construcción. Tampoco encontramos en este autor referencia alguna sobre las implicaciones de utilizar números reales para expresar los precios, es decir para la medida económica de las mercancías.

Debreu busca mantener simultáneamente dos elementos que son incompatibles: por un lado, la idea de que existe una operación de medida económica (a los bienes económicos se les asigna un número real que es su precio); por el otro, las mercancías objeto de esta operación de medida son los bienes físicamente especificados y heterogéneos. Precisamente, aquí yace el núcleo de la contradicción: en tanto valores de uso, los bienes físicos pueden guardar sus dimensiones heterogéneas, pero las mercancías, en tanto objeto de una operación de medida, son magnitudes homogéneas. Como el único referente que propone Debreu para las "mercancías" son los objetos físicos, el resultado es la indeterminación del concepto central de la teoría.

números reales, tanto para las cantidades de los bienes físicos, como para los precios, ya que las raíces de las ecuaciones características pueden ser elementos de $R$. 


\section{Apéndice*}

\section{NOTAS SOBRE LA TEORÍA DE LA MEDIDA}

A continuación se presentan algunos de los elementos más importantes de la teoría de la medida que deben ser considerados en el estudio de la teoría del valor. El punto de partida es la definición convencional de la medida; tomamos la ofrecida por Doneddu (1971:228-229):

La medida de una magnitud es definida como la correspondencia a todo elemento $x_{i}$ de $X$ de un número real positivo y uno solo, denominado medida de $x_{i}$ y denotado por $v\left(x_{i}\right)$ de tal forma que a un elemento normal $u$ de $X$ y escogido de una sola vez y llamado "unidad de medida" le corresponde el

* Las notas que aquí se presentan reúnen, en primer lugar, a los elementos más importantes que permiten caracterizar a la teoría de la medida de acuerdo a Félix (1966), Lebesgue (1975) y Nivollet (1981). En segundo lugar, se exponen las principales implicaciones de concebir al intercambio como una operación de medida de acuerdo a los trabajos fundamentales de Fradin (1973) y Nivollet (1980). 
número 1 y que a la agregación de dos elementos de $X$ corresponde la suma de sus medidas.

Aquí encontramos la distinción entre "magnitud" y "medida de la magnitud". Una magnitud es lo que constituye el objeto de la operación de medida: y para evitar entrar en discusiones de carácter metafísico, Lebesgue (1975) señala que se trata de un concepto cuya esencia reside en las operaciones que definen su medida, de tal manera que el concepto cuantitativo se elabora a partir de los procedimientos de medida.

En la definición anterior podemos encontrar los elementos esenciales de la base axiomática de la teoría de la medida. En primer lugar, tenemos la presencia de los axiomas de orden indicando que cualquier orden inducido sobre los objetos medidos por la operación de medida debe ser igual al orden derivado de la operación empírica inicial. ${ }^{1}$ En segundo lugar, la operación de medida no sólo debe preservar el orden, sino que debe ser aditiva en el sentido siguiente: la asociación de un número a los objetos debe ser una representación "extensiva" del ordenamiento empírico. De esta manera, y de acuerdo con la definición de Doneddu, tenemos las relaciones siguientes:

$$
\begin{aligned}
& v(u)=1 \\
& v\left(x_{i} \oplus x_{j}\right)=v\left(x_{i}\right)+v\left(x_{j}\right)
\end{aligned}
$$

en donde $u, x_{i}$ y $x_{i}$ son magnitudes de la misma especie que son objeto de una operación de medida. Aquí, la operación $\oplus$ significa "concatenación" o "agregación" y está definida en el ordenamiento empírico. La propiedad básica de la concatenación es que es débilmente conmutativa y débilmente asociativa; las dos propiedades están ligadas por la de la monotonicidad débil (el orden empírico $a \gtrsim b$ se mantiene si, y sólo si, el orden de la concatenación con otro objeto $c$, $a \oplus c \gtrsim b \oplus c$ también se mantiene).

Tomando en cuenta estos elementos, es posible reformular la definición anterior como sigue: la medida de una magnitud es el

1 En este sentido, el primer problema que la teoría de la medida debe resolver es el de la caracterización de las propiedades formales 0 abstractas de los procedimientos empíricos y observaciones que dan origen a las magnitudes cuantificables. Después debe buscar mostrar cómo la asignación de números (involucrada en la operación de medida) es de tal forma que las relaciones abstractas (por ejemplo, las del orden $\geq 0$ la de la suma + ) corresponden estructuralmente a las relaciones empíricas y concretas. 
coeficiente numérico por el cual hay que multiplicar una otra magnitud de la misma especie, denominada unidad de medida,para formar la primera magnitud. Tenemos así que la medida de $x_{i}$ es el número $\alpha$ tal que $x_{i}=\alpha \cdot \mu$ (en donde $x_{i}$ y $u$ pertenecen al conjunto de magnitudes, y $\alpha \in R$ ).

El espacio de medidas puede ser definido de acuerdo a ciertas propiedades que sean juzgadas como pertinentes. Como ya se indicó, el postular estas propiedades condiciona la manera en que se construye el concepto de la magnitud objeto de la operación de medida (Lebesgue, 1975). Por diversos tipos de razones que no se analizan aquí, la teoría económica ha establecido las propiedades siguientes para el espacio de medidas. En primer lugar, dicho espacio tiene la estructura de un semigrupo abeliano: se trata de un conjunto dotado de una ley de composición interna asociativa y conmutativa, para la cual no existen elementos simétricos en el conjunto de medidas. También está dotado de un elemento neutro y de un orden total.

En segundo lugar, se postula que el espacio de medidas deberá tener las siguientes propiedades: ser completo y arquimediano. Por el hecho de ser completo, el espacio de medidas no tiene lagunas y toda magnitud va a tener una medida, cualquiera que sea la unidad de medida escogida. Por definición, el espacio es completo si para cualquier sucesión de Cauchy de elementos de ese espacio, dicha sucesión es convergente y su punto límite se encuentra en el espacio: sea $A$ un espacio cualquiera y $\quad\left\{x_{i}\right\}_{i=1}$ una sucesión en ese espacio; dicha sucesión es de Cauchy si dado

$$
\epsilon>0, \exists n_{o} \in N \text { tal que } \forall n, p \in N \text { con } n \geqslant n_{o} \text { y } p \geqslant n_{o}
$$

entonces

$$
d\left(x_{n}, x_{p}\right) \leqslant \epsilon
$$

el espacio $\boldsymbol{A}$ es completo si contiene el límite $\boldsymbol{e}$ de esta sucesión:

$$
\forall \epsilon>O, \exists n \in N, \forall n_{o} \geqslant n, d\left(x_{n_{o}}, e\right) \leqslant \epsilon, \text { у } е \epsilon A .
$$

Esto significa que el conjunto de los números reales contiene la totalidad de sus propios puntos límites: en la terminología de Dantzig, es "perfecto". Por el hecho de ser completo, dicho espacio no es numerable. 
La definición de espacio completo implica la noción de distancia: cualesquiera que sean dos medidas definidas en ese espacio, existe siempre una tercera medida en el intervalo que las separa (aunque éste sea arbitrariamente pequeño) y se demuestra que existe una infinidad. ${ }^{2}$ Esta ausencia de lagunas o de intervalos vacíos es la característica del continuum y es la base del axioma Cantor-Dedekind para la medida real: ${ }^{3}$

Es posible asignar a cualquier punto de una línea un número real único $y$, recíprocamente, cualquier número real puede ser representado de manera única por un punto de una línea.

Aquí la realidad ontológica de la "línea recta" se transforma y lo único que queda es un conjunto isomorfo a $R$; la "recta de los reales" no es más que un vehículo de los números reales (Dantzig). El alejamiento del mundo empírico y del de la realidad ontológica de cada punto elemento de una línea, no puede ser más radical.

Por otra parte, la propiedad arquimediana o de divisibilidad perfecta implica que por más pequeña que sea una medida, y por más grande que sea otra, siempre existe un múltiplo de la primera que es superior a la segunda. En otros términos, sean $\alpha$ y $\beta$ dos elementos del espacio de medida $M$, con $\alpha>\beta$; entonces $\exists n \in M$ tal que $n$ $\beta>\alpha$. El corolario es que existe $n \in M$ tal que $1 / n \alpha<\beta$. Esto significa que toda medida es divisible.

Con estas propiedades se garantiza que toda magnitud tenga una medida, y que tanto $\alpha$ como $\beta$ puedan ser números irracionales. Se está entonces postulando que el espacio de medida es isomorfo a $R$. El procedimiento para construir una medida real implica que el espacio de magnitudes sea isomorfo al espacio de medida, y por lo tanto a $R$.

Se puede proseguir ahora con el examen de la concepción del intercambio como una operación de medida a través de la relación de equivalencia. El objetivo es contrastar el procedimiento de homogenización que subyace a esta operación con el procedimiento presentado por Debreu. El punto de partida es el siguiente: sobre el conjunto de mercancías se puede definir una relación de equivalencia $L$, expresión de la relación de intercambio del tipo $x_{i} \stackrel{\leftrightarrow}{\rightarrow} x_{j} \Rightarrow x_{i} L x_{j}$. De esta manera, $L$ es un subconjunto de $L \subset \hat{E} \times \hat{E}$ tal que verifique las siguientes propiedades:

2 En este contexto, $d$ satisface las propiedades de toda métrica.

3 Citado por Dantzig (1974:180). 
1) $a \in \hat{E},(a, a) \in L$; (reflexividad)

2) si $(a, b) \in L$, entonces $(b, a) \in L$; (simetría)

3) $\operatorname{si}(a, b) \in L,(b, c) \in L$, entonces $(a, c) \in L$; (transitividad)

Con esta relación de equivalencia se define un conjunto cociente.

$$
\hat{E} / L=\left\{c_{\alpha}, C_{\beta}, \ldots,\right\}
$$

integrado por las clases de equivalencia formadas sobre los elementos de $E$ por $L$. Así la relación de intercambio permite ubicar a todos los bienes de $\hat{E}$ en el interior de clases de equivalencia: si se verifica $x_{i} \rightleftarrows x_{j}$ entonces $x_{i} L x_{j}$ y podemos afirmar que ambos elementos pertenecen a la misma clase de equivalencia. Por definición, las clases de equivalencia están desconectadas de tal forma que cada elemento de $\hat{E}$ se encuentra en el interior de una sola clase de equivalencia.

Por otra parte, la partición de $\hat{E}$ por $L$ permite llevar a cabo una operación de abstracción, pues una relación de equivalencia sólo es inteligible cuando los objetos en cuestión tienen la misma propiedad. A cada clase de equivalencia le está asociado un coeficiente $\alpha$, que constituye su "precio". ${ }^{4}$ Ese coeficiente no es otra cosa que el valor económico de los bienes involucrados en la relación de equivalencia puesto que $x_{i} L x_{j}$ implica que $v\left(x_{i}\right)=v\left(x_{j}\right)$. Esto significa simplemente que los bienes que se intercambian tienen la misma medida. Para que este enunciado tenga un sentido preciso se requiere haber definido la unidad de medida (i.e., la dimensión). Los bienes $x_{i}$ y $x_{j}$ no son iguales en tanto bienes físicos, sino en tanto equivalentes (es solamente desde este punto de vista que cualquier elemento de una clase de equivalencia es apto para representar a los demás). En consecuencia, lo que forma a las clases de equivalencia, o los elementos que la integran, son magnitudes conmensurables y la unión de todas ellas permite definir al conjunto cociente.

Precisemos este punto. Para poder concebir al intercambio como relación de equivalencia, es necesario reducir los objetos que en él intervienen a especies de un mismo espacio (Marx). Así, aunque aparentemente se intercambian $x_{i} \rightleftarrows x_{j}$, para la teoria la relación se

4 La partición de $\hat{E}$ por $L$ corresponde a una operación de abstracción matemática: el valor o coeficiente asociado a cada clase no es más que un elemento abstracto, caracterizado por la propiedad común a todos los elementos pertenecientes a dicha clase de equivalencia. Sobre la naturaleza de la operación de abstracción matemática, véase Vuillemin (1971). 
expresa como $v\left(x_{i}\right)=v\left(x_{j}\right)$. Habiéndose llevado a cabo una operación de abstracción por la relación de equivalencia, se concluye que lo que entra en las clases de equivalencia ya no son los objetos físicos iniciales, sino ciertas cantidades de magnitudes homogéneas (Félix). Así, es posible construir las clases de equivalencia, pero sólo a condición de abandonar el mundo de los objetos físicos. En consecuencia, lo que forma parte de las clases de equivalencia son magnitudes homogéneas (i.e., cantidades de la mercancía) que son el objeto de una medida a través de la operación de intercambio. ${ }^{5}$

\section{Bibliografía}

1. BenetTI, C. y J. CARTElier, Marchands, salariat et capitalistes, Paris, Maspero, 1980.

2. Dantzig, T., Le nombre: language de la science, Paris, A. Blan chard, 1974.

3. DebreU, G., Theory of Value (An Axiomatic Analysis of Economic Equilibrium), Yale University Press, 1959. (Existe traducción al español: Teoria del Valor, Antoni Bosch, Barcelona, 1973).

4. DONEDDU, A., Les bases de l'analyse mathématique moderne, Paris, Dunod, 1971.

5. FÉLIX, L., Modern Mathematics and the Theacher, Cambridge University Press, 1966.

6. Fradin, J., Valeur, monnaie et capital, Tesis doctoral, Universidad de Paris I, 1973.

7. Koopmans, T.C. y A.F. Bausch, "Selected Topics in Economics Involving Mathematical Reasoning", SIAM Review, vol. I, No. 2, July 1959.

8. Lebesgue, H., La mésure des grandeurs, Paris, A. Blanchard, 1975.

9. Malinvaud, E., Leçons de théorie microeconomique, Paris, Dunod, 1979.

5 Lucienne Félix, asociada al grupo Bourbaki, preocupada por las confusiones que se insinúan en el manejo de la teoría de conjuntos, señala: "La relación de equivalencia implica que los objetos tienen la misma propiedad o pertenecen a la misma clase. En el primer caso suponemos conocida la propiedad de que se está hablando y que permite definir a la relación de equivalencia. En el segundo caso, se supone que el conjunto de referencia ya ha sido subdividido en clases y que esta división es la base de la definición de equivalencia." Véase Félix (1960). 
10. MARx, K., Théories sur la plus-value, Paris, Editions Sociales, 1976 (Tomo III).

, Contribution a la critique de l'économie politique, Paris, Editions Sociales, 1977.

11. NAdAl, A., "Dinero y valor de uso: la noción de riqueza en la génesis de la economía política", El Colegio de México, Serie de Documentos de Trabajo del CEE, No. XI, 1983.

12. Nivollet, B., La monnaie: essai sur la détermination d'un concept economique, Tesis doctoral, Universidad de Paris I, 1980.

13. Ricardo, D., Principles of Political Economy and Taxation (1821) en Works and Correspondence of David Ricardo, Vol. I (Edición de P. Sraffa), Cambridge University Press, 1951.

14. SAlAS, C. y A. VALLE, "La relación economía-matemáticas en Piero Sraffa", Ensayo presentado en el Coloquio de Economía y Matemáticas (IIMAS-CCH), diciembre, 1981.

15. Vuillemin, J., La logique et le monde sensible, Paris, Flammarion, 1971. 